\title{
Application of ionospheric corrections in the equatorial region for L1 GPS users
}

\author{
Paulo de Oliveria Camargo ${ }^{1}$, João Francisco Galera Monico ${ }^{1}$, and Luiz Danilo Damasceno Ferreira ${ }^{2}$ \\ ${ }^{1}$ Department of Cartography, São Paulo State University, Rua Roberto Simonsen, 305 Centro Educacional, \\ 19060-900 Presidente Prudente, SP, Brazil \\ ${ }^{2}$ Department of Geomatic, Paraná Federal University, Centro Politécnico - Jardim das Américas, 81531-990 Curitiba, PR, Brazil
}

(Received January 7, 2000; Revised September 21, 2000; Accepted September 21, 2000)

\begin{abstract}
In the absence of the selective availability, which was turned off on May 1, 2000, the ionosphere can be the largest source of error in GPS positioning and navigation. Its effects on GPS observable cause a code delays and phase advances. The magnitude of this error is affected by the local time of the day, season, solar cycle, geographical location of the receiver and Earth's magnetic field. As it is well known, the ionosphere is the main drawback for high accuracy positioning, when using single frequency receivers, either for point positioning or relative positioning of medium and long baselines. The ionosphere effects were investigated in the determination of point positioning and relative positioning using single frequency data. A model represented by a Fourier series type was implemented and the parameters were estimated from data collected at the active stations of RBMC (Brazilian Network for Continuous Monitoring of GPS satellites). The data input were the pseudorange observables filtered by the carrier phase. Quality control was implemented in order to analyse the adjustment and to validate the significance of the estimated parameters. Experiments were carried out in the equatorial region, using data collected from dual frequency receivers. In order to validate the model, the estimated values were compared with "ground truth". For point and relative positioning of baselines of approximately $100 \mathrm{~km}$, the values of the discrepancies indicated an error reduction better than $80 \%$ and $50 \%$ respectively, compared to the processing without the ionospheric model. These results give an indication that more research has to be done in order to provide support to the L1 GPS users in the Equatorial region.
\end{abstract}

\section{Introduction}

The main sources of systematic errors that affect the positioning with Global Positioning System (GPS) are associated to the satellite (orbit error, clock bias, relativity, group delay), to the propagation of the sign (troposphere and ionosphere refraction, cycle slips, multipath), to the receiver/antenna (clock bias, interchannel bias, antenna phase center) and to the station (coordinates, earth body tides, polar motion, ocean tide loading, atmosphere pressure loading) (Monico, 1995). These errors can be modeled or reduced if appropriate tracking and processing techniques are adopted.

In the absence of the limitation imposed to the civil users by the United State Department of Defense (DoD), through the adoption of the Selective Availability (SA), implemented intentionally in the system during the period of July 4, 1994 to May 1, 2000, the ionosphere can be the largest source of error in the positioning with single frequency GPS receivers. It affects directly the point positioning technique, while in the relative positioning of short baselines, such effects are practically eliminated. The error due to the temporary ionosphere behavior depends on several variables, such as time of the day, season, solar cycle, geographical location of the observer and Earth's magnetic field, and is difficult to be corrected. During the maximum solar activity and for satellite

Copy right (c) The Society of Geomagnetism and Earth, Planetary and Space Sciences (SGEPSS); The Seismological Society of Japan; The Volcanological Society of Japan; The Geodetic Society of Japan; The Japanese Society for Planetary Sciences. close to the horizon, it can be larger than 100 meters (Newby and Langley, 1992).

Dual frequency GPS receivers allow correction of the ionospheric refraction effects, providing results that are practically free of such effects, for data collected during time period of minimum solar activity. However, as such receivers are very expensive, the single frequencies ones are used extensively in the determination of baselines, even in conditions, which are not totally appropriate. When using these receivers, however, the ionospheric systematic effect deteriorates surveying results for medium and long baselines. The same can be said for point positioning. However, for short baselines, with distances smaller than $10 \mathrm{~km}$, the relative positioning can be mostly accurate (Wells et al., 1986).

The navigation messages transmitted by the GPS satellites contain information that allows correcting single frequency GPS receiver observables by using the Klobuchar model (Klobuchar, 1987). Several studies showed this model can remove just around $50 \%$ of the total effect (Newby and Langley, 1992). Therefore, it is necessary to have a more effective way of eliminating such effects, which can be investigated from the use of regional and local ionospheric models.

Studies related to the use of GPS in the south of Brazil and in the equatorial region showed that in Central America and in South America the GPS observables are affected by severe ionospheric conditions (Wanninger et al., 1991, 1993; Campos et al., 1993). These conclusions were obtained from 


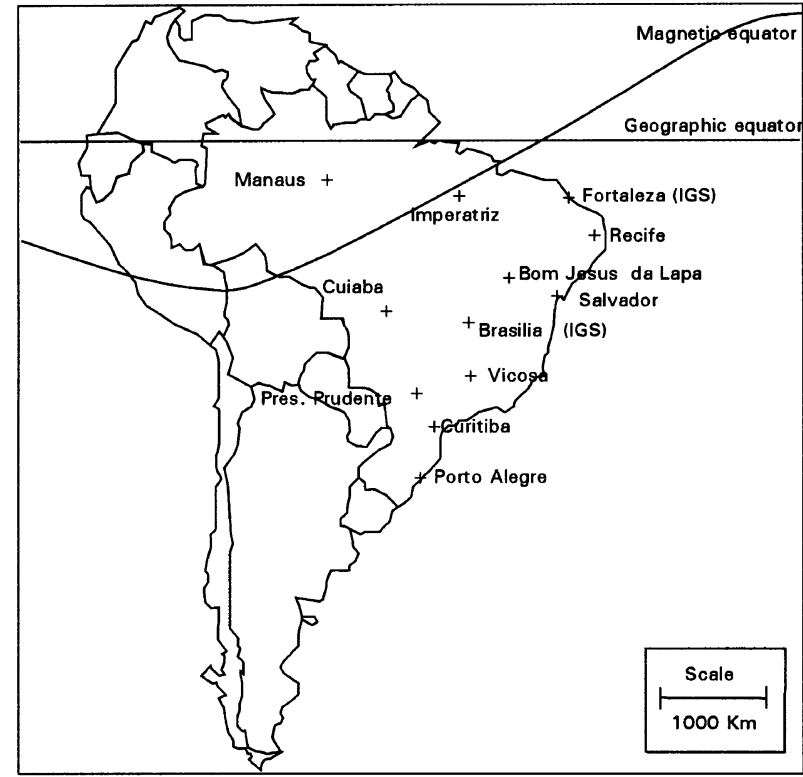

Fig. 1. RBMC stations - Brazil.

results of two campaigns, called GPS BRASION'91 (BrasilIonosphere) and BRASION'92. The regions of highest ionospheric delay are located, on average, approximately $\pm 15^{\circ}$ to $\pm 20^{\circ}$ on either side of the earth's geomagnetic equator (Klobuchar, 1991).

With the establishment of the Brazilian Network for Continuous Monitoring of GPS satellites (RBMC) by the Fundação Instituto Brasileiro de Geografia e Estatística (IBGE), a very large data base becomes available to accomplish studies related to the ionosphere in Brazilian conditions. Nowadays, RBMC is composed of 12 stations, collecting GPS data continuously. Two of these stations, in Brasília and in Fortaleza, make part of IGS (International GPS Service) network. One of the main objectives of RBMC is its use as a reference for relative positioning. The network operates with dual frequency GPS receivers, except in the Fortaleza station, where a Rogue SNR-8000 receiver operates, the other stations are equipped with Trimble 4000 SSI. They are located in Bom Jesus da Lapa/BA (BOMJ), Brasília/DF (BRAZ), Cuiabá/MT (CUIB), Curitiba/PR (PARA), Fortaleza/CE (FORT), Imperatriz/MA (IMPZ), Manaus/AM (MANA), Presidente Prudente/SP (UEPP) and Viçosa/MG (VICO), Porto Alegre/RS (PORT), Recife/PE (RECF) and Salvador/BA (SALV) (Fig. 1).

The aim of this work is to define and establish a mathematical model that represents the Brazilian ionospheric conditions in order to provide capability to the single frequency GPS users to correct theirs observables from such effects. Data from RBMC provide the input to the model.

\section{Ionosphere}

Considering the propagation aspects of the GPS signals, it is convenient to subdivide the atmosphere in troposphere and ionosphere, because the conditions of signal propagation are different for each one. The layers closer to the earth atmosphere are called troposphere, which extends from the earth's surface to about $50 \mathrm{~km}$ above the earth. It constitutes the neutral zone of the atmosphere, and the propagation of the signal depends mainly on the water vapor content, the air pressure and the temperature of the atmospheric layers. In this case, the refraction is independent of the signal frequency, since its frequency is below $30 \mathrm{GHz}$ (Leick, 1995). The ionosphere covers a region between $50 \mathrm{~km}$ and $1000 \mathrm{~km}$ and is characterized by a significant number of free (negatively charged) electrons and positively charged atoms and molecules called ions. In this region the signal propagation depends on the frequency.

The GPS signals, on their path between satellites and receivers, propagate through the dynamic atmosphere, and thus experience different kinds of influences. Variations may occur in the direction of propagation, in the velocity of propagation and in the signal strength. The ionosphere is a dispersive medium, meaning that the modulation on the carrier and the carrier phase are affected differently and that this effect is a function of carrier frequency. Therefore, these effects cause an increase and a decrease in the distances obtained from the code and carrier phase, but of the equal magnitude. Since the ionospheric effect depends on the frequency, consequently it depends on the refraction index as well. It is proportional to the total electron content (TEC), the number of electrons present along the path of the signal between the satellite and the receiver. The main problem is that TEC varies in time and space.

The refraction index of the phase $(+)$ and group $(-)$, considering only the first order effects, is given by:

$$
n_{f}=1 \pm \frac{40.3 n_{e}}{f^{2}}
$$

where $n_{e}$ is the electron density, which is given in units of electrons per cubic meter and $f$ is the frequency of the signal.

For group delay of GPS signal arriving at station $(r)$ from satellite $(s)$, the ionospheric refraction $\left(I_{r}^{S}\right)$ is given by (Leick, 1995):

$$
I_{r}^{s}=\frac{40.3}{f^{2}} \mathrm{TEC} .
$$

\section{Ionospheric Correction for Positioning with Single Receivers}

Some methods have been used to determine the systematic effect due to ionospheric refraction in the L1 carrier of single frequency GPS receivers. The quantification of this effect can be evaluated by:

- Coefficients transmitted in the navigation messagesKlobuchar model;

- Observables collected with single frequency GPS receivers;

- Observables collected with dual frequency GPS receivers.

In this work the method that uses data from dual frequency, especially the pseudorange filtered by the carrier phase will be focused. In the derivation of the model, errors due to nonsynchronism of the satellite and receiver clocks, ephemerides and the tropospheric refraction will be neglected, since their effects contaminate both frequencies in the same way and do not affect the validity of the results. The model is based on the difference between pseudoranges $\left(P_{r}^{s}\right)$ of the carriers L2 


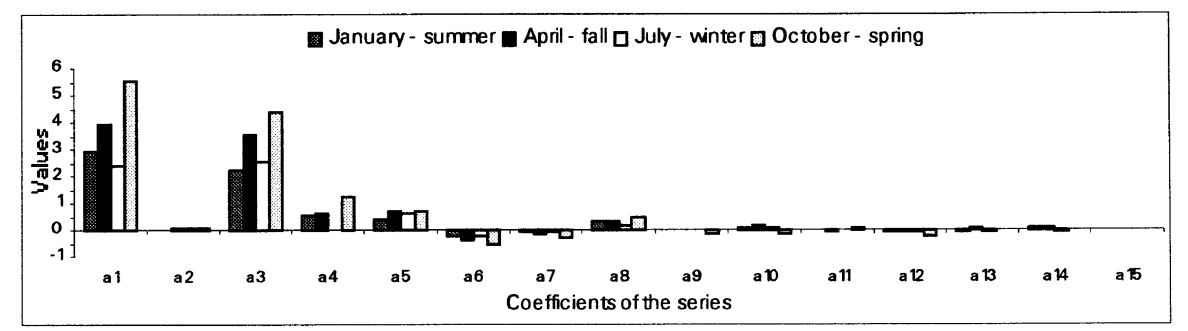

Fig. 2. Estimated average coefficients of the model.

and L1. It is given by (Georgiadiou, 1994):

$$
P_{2 r}^{s}-P_{1 r}^{s}=I_{2 r}^{s}-I_{1 r}^{s}+\left(S_{p 2}^{s}-S_{p 1}^{s}\right)+\left(R_{p 2}-R_{p 1}\right)+\epsilon_{p 21} .
$$

Using Eq. (2) gives:

$$
\begin{aligned}
I_{2 r}^{s}-I_{1 r}^{s} & =40.3 \operatorname{TEC}^{s} \frac{f_{1}^{2}-f_{2}^{2}}{f_{1}^{2} f_{2}^{2}} \\
& =I_{1 r}^{s} \frac{f_{1}^{2}-f_{2}^{2}}{f_{2}^{2}}=I_{1 r}^{s} \frac{1}{F},
\end{aligned}
$$

thus:

$$
\begin{aligned}
F\left(P_{2 r}^{s}-P_{1 r}^{s}\right)= & I_{1 r}^{s}+F\left[\left(S_{p 2}^{s}-S_{p 1}^{s}\right)+\left(R_{p 2}-R_{p 1}\right)\right] \\
& +F \epsilon_{p 21} .
\end{aligned}
$$

This equation is used to estimate the ionospheric slant delay $\left(I_{1 r}^{s}\right)$ in the L1 carrier, based on pseudorange observables. The differences $\left(S_{p 2}^{s}-S_{p 1}^{s}\right)$ and $\left(R_{p 2}-R_{p 1}\right)$ represent, respectively, L1-L2 satellite and receivers interfrequency biases, and $\epsilon_{p 21}$ represents another differential errors.

The model developed by Georgiadiou (1994) for modeling the ionosphere was a contribution towards the development of a regional model based on GPS for the area covered by the Active GPS Reference System (AGRS) of the Netherlands. It consists of a modification of the model developed by Georgiadiou and Kleusberg (1988), to calculate the ionospheric L1 delay:

$$
I_{1 r}^{s}=\frac{I_{1}^{v}}{\cos \left(z^{\prime s}\right)},
$$

where $I_{1}^{v}$ represents the vertical ionospheric delay and $z^{\prime s}$ the zenital angle of the signal path from the satellite $(s)$ to a point (ionospheric point) in an ionospheric layer of $400 \mathrm{~km}$ of height. Thus:

$$
\begin{aligned}
F\left(P_{2 r}^{s}-P_{1 r}^{s}\right)= & \frac{I_{1}^{v}}{\cos \left(z^{\prime s}\right)}+F\left[\left(S_{p 2}^{s}-S_{p 1}^{s}\right)+\left(R_{p 2}-R_{p 1}\right)\right] \\
& +F \epsilon_{p 21} .
\end{aligned}
$$

The term on the left side of this equation represents the ionospheric delay in the L1 carrier, obtained from the measurements of the pseudoranges in both frequencies. Georgiadiou (1994) used the following series to represent the diurnal behavior of vertical ionospheric delay:

$$
\begin{aligned}
I_{1}^{v}= & a_{1}+a_{2} B^{s}+\sum_{\substack{i=1 \\
j=2 i+1}}^{n}\left\{a_{j} \cos \left(i h^{s}\right)+a_{j+1} \sin \left(i h^{s}\right)\right\} \\
& +a_{n * 2+3} B^{s} h^{s}
\end{aligned}
$$

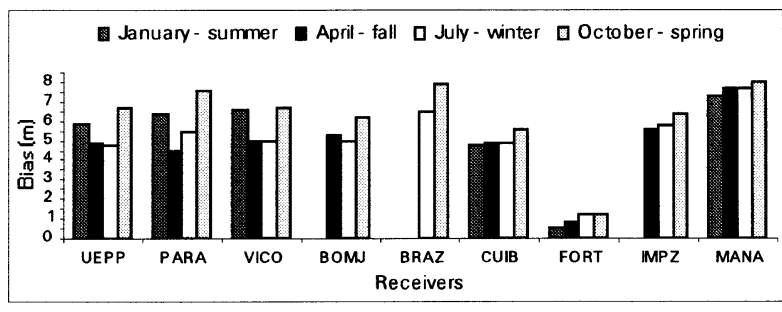

Fig. 3. L1-L2 interfrequency bias average due to the receivers.

where the value of $n$ depends on the significance of the parameter.

The variable $B^{s}$ is the difference between the receiver latitude and the latitude of the subionospheric point (projection of a point on ionospheric layer upon the earth surface). The variable $h^{s}$ is given as:

$$
h^{s}=\frac{2 \pi}{T}\left(t-14^{h}\right),
$$

where $T$ represents the period of 24 hours and $t$ is the local time in hours, of the subionospheric point.

In the Georgiadiou (1994) experiments, only one receiver was used and the groups L1-L2 of the interfrequency biases $\left(C^{s}=F\left[\left(S_{p 2}^{S}-S_{p 1}^{s}\right)+\left(R_{p 2}-R_{p 1}\right)\right]\right)$ were estimated for each satellite. In our experiment, where several receivers of RBMC were involved, the interfrequency biases of the satellite and the receivers were estimated separately. The total number of unknown parameters are $15+r+s$, where 15 represent the coefficients of the series, $r$ corresponds to the L1-L2 receivers interfrequency biases, which is equal to the number of network stations and $s$ corresponds to the L1L2 satellite interfrequency biases, being equal to the number of tracked satellites. The number of equations available is greater than the number of unknown parameters, enabling to apply the least-squares method. The design matrix A presents rank deficiency, equal to one (Camargo, 1999). Therefore, the L1-L2 receivers or satellites interfrequency biases have to be determined in relation to one of them.

\section{Experiments}

A software in Lahey FORTRAN 95 was implemented in order to estimate the parameters of the proposed model. These parameters were estimated through the least-squares adjustment using the method of the observation equations with constraints. Quality control based on the Chi-square statistical test $\left(\chi^{2}\right)$ was implemented for the adjustment anal- 


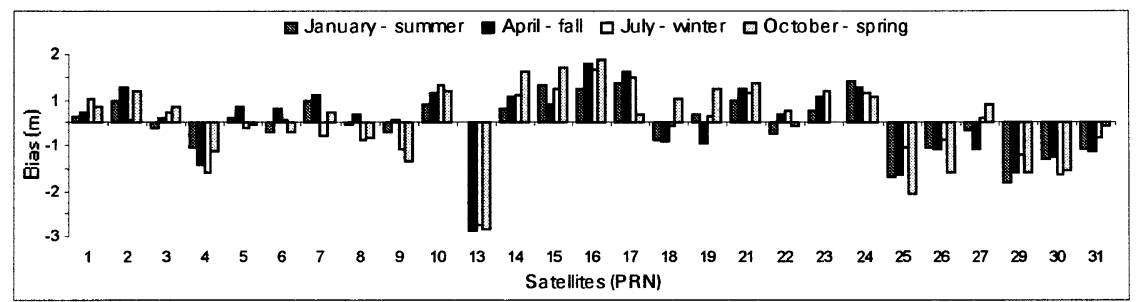

Fig. 4. L1-L2 interfrequency bias average due to the satellites.

ysis (Teunissen, 1985), as well as a test for the significance of the parameters used in the model (Zhong, 1997). The software allows estimating the coefficients of the model and can provide corrections to the L1 carrier observables. The observation files used to calculate the coefficients, as well as the ones corrected must be in the RINEX format. Therefore, the data processing can be carried out by any ordinary GPS software that accepts such a format.

In the experiments, data from 9 RBMC stations were used, with 30 seconds data rate. The ionosphere layer adopted was $400 \mathrm{~km}$, and the observables used were the pseudorange filtered by the carrier phase, using the algorithm presented by Jin (1996). The filtering was used with the aim of handling the multipath error and the noise presented in the pseudoranges observations. Only data collected above 15 degrees of elevation were used.

Tests with point positioning and relative positioning were accomplished. For the former, the data were processed with the software GPSPACE Version 3.2, developed by the Geodetic Survey Division, Natural Resources Canada (NRCan, 1997). The baselines data used in the analysis were processed using the software GPSurvey Version 2.2 of Trimble.

\subsection{Model parameter analysis}

An analysis of the parameters of the model will be presented in this section. The data used refer to four months of 1998, enclosing one-month of each four seasons.

In order to remove the L1 systematic effects of the reference station, the average of the interfrequency biases of the satellites was calculated $\left(\left\langle F\left(S_{p 2}^{s}-S_{p 1}^{s}\right)\right\rangle\right)$ and it was subtracted from the individual daily value of each satellite, because the average variation reflects the variation of the reference station (Sardon and Zarraoa, 1997). The interfrequency biases of each satellite relative to the average are given by:

$$
F\left(S_{p 2}^{s}-S_{p 1}^{s}\right)^{m}=F\left(S_{p 2}^{s}-S_{p 1}^{s}\right)-\left\langle F\left(S_{p 2}^{s}-S_{p 1}^{s}\right)\right\rangle .
$$

Therefore, the interfrequency bias due to the receivers, relative to the average, is calculated as:

$$
F\left(R_{p 2}-R_{p 1}\right)_{r}^{m}=F\left(R_{p 2}-R_{p 1}\right)_{r}-\left\langle F\left(S_{p 2}^{S}-S_{p 1}^{s}\right)\right\rangle .
$$

This procedure, according to Sardon and Zarraoa (1997), removes the effect of the interfrequency bias of the reference station, but it does not make possible to obtain the absolute interfrequency biases of the satellites or the receivers in L1. Such values, related to the satellites, are transmitted in the broadcast messages. From April 1999, they are providing by JPL (Jet Propulsion Laboratory), since the values supplied

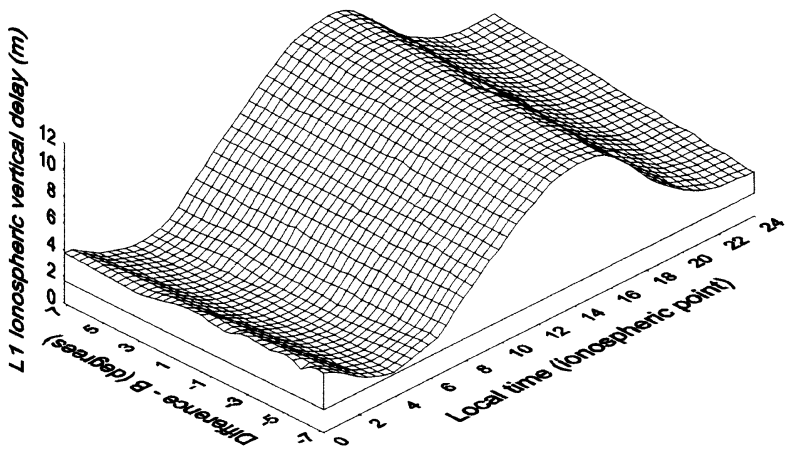

Fig. 5. Estimated Ionospheric vertical delays - UEPP station (274/1998).

by the satellite maker presented some problems (Wilson et al., 1999).

Figures 2, 3 and 4 show, respectively, the coefficient averages estimated from the model, the systematic L1 error due to the receivers and due to the satellites. These values represent the average estimated for each month.

The coefficients shown in Fig. 2, mainly $\mathrm{a}_{1}$ and $\mathrm{a}_{3}$, provide an idea of the ionosphere seasonal behavior. There is a high ionosphere activity in the months of April (fall) and October (spring), soon after the equinoxes, while it is smaller in January (summer) and July (winter), after the solstices. The precisions $(1 \sigma)$ of the daily coefficients averages were better than $0.019 \mathrm{~m}$ and for the monthly coefficients averages were better than $0.753 \mathrm{~m}$. In the accomplished experiments, the order of the series given by Eq. (8), with $n=6$, was appropriated. The significance test of the parameters showed that in $61 \%$ of the experiments the coefficients $a_{13}$ and $a_{14}$ were not significant.

The maximum value of the receivers interfrequency biases occurred in January and October and the minimum in April and July (Fig. 3). The precisions of the daily and monthly averages were better than $0.027 \mathrm{~m}$ and $0.848 \mathrm{~m}$. Concerning the systematic error of the satellites (Fig. 4), the individual average of each satellite showed a random behavior. The largest discrepancies mainly occurred in October. The daily and monthly precisions averages for L1-L2 satellites interfrequency biases were, respectively, better than $0.061 \mathrm{~m}$ and $0.662 \mathrm{~m}$.

\subsection{Ionospheric vertical delay}

With the aim of showing the systematic error behavior due to the ionosphere for UEPP station, during a 24-hour period, Fig. 5 is present. It refers to October 1, 1998, month that presented maximum ionospheric effects. It shows the 

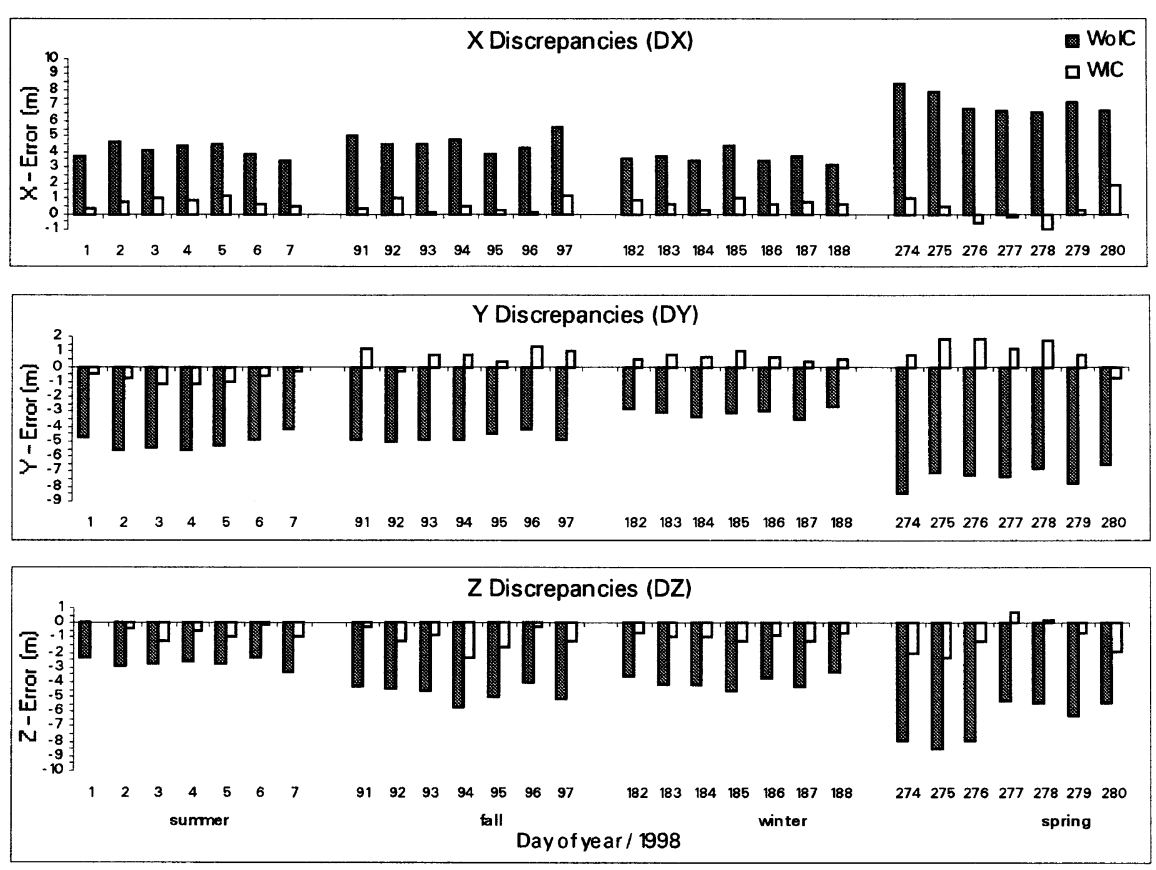

Fig. 6. Discrepancies between the "true" and the estimated cartesian coordinates of UEPP station (WoIC and WIC refer to the solution without and with ionospheric corrections).

ionospheric vertical delay resulting from the adopted model, as a function of the local time of the ionospheric point and of the difference (B) between the station latitude and the latitude of the sub-ionospheric point.

From Fig. 5 it can be observed that the daily maximum ionosphere activity occurs close to the 14:00 local time, as expected. The ionospheric quality model can be verified with a residual analysis, applying the GOM (Global Overall Model) (Teunissen, 1985). They presented average close to zero and standard deviation of $1.5 \mathrm{~m}$, except in October, when the standard deviation was about $2.2 \mathrm{~m}$. Based on the GOM statistic, the model was accepted with significance level $\alpha$ equal to $5 \%$.

\subsection{Point positioning test}

The quality of the implemented model can be analysed by comparing the results obtained with the adopted model, against a considered "ground truth". As "ground truth", the SIRGAS (Geocentric System of Reference to South America) coordinates of the UEPP station were considered. It is worth to mention that the UEPP station did not participate in the group of stations that provided data to estimate the parameters of the model. Therefore, it provides an independent result.

For point positioning from pseudorange observables (C/A), the data of the UEPP station during 1998 were used, enclosing one week of each seasons. The values estimated for the cartesian coordinates $(X, Y, Z)$ were daily compared with corresponding the "ground truth". It was considered the cases without ionospheric correction (WoIC) and with ionospheric correction (WIC).

The precise ephemerides and clock corrections of the satellites produced by GSD/NRCan were used. The objective was to eliminate the effects of SA that is the largest error source in the point positioning. In order to avoid estimated positions with poor geometry, only GDOP (geometric dilution precision) equal or smaller than 7 was used. In the processing, only pseudorange collected above 15 elevation degrees was considered, and the precision adopted was of $3.0 \mathrm{~m}$. The meteorological data used for tropospheric corrections were collected in the FCT/Unesp meteorological station, close to the UEPP station.

Figure 6 shows the discrepancies among the considered "true" cartesian coordinates of the UEPP station and the estimated ones (WoIC and WIC) for a 24-hour period.

The mean discrepancies for the processing WIC indicated discrepancies with respect to the ground truth better than a meter. Considering the resulting error $\left(\sqrt{\left(D X^{2}+D Y^{2}+D Z^{2}\right.}\right)$, there was an error reduction of about $80 \%$. It represents a mean error reduction from $8.44 \mathrm{~m}$ to $1.61 \mathrm{~m}$. While the maximum error WoIC was $14.21 \mathrm{~m}$, it was reduced to $5.33 \mathrm{~m}$ in the WIC processing. Considering the minimum error, the reduction was from $2.96 \mathrm{~m}$ (WoIC) to $0.55 \mathrm{~m}$ (WIC).

The discrepancies in the results obtained in an epoch per epoch solution for the first day of October are shown in Fig. 7.

In the epoch per epoch solution, the results present the diurnal behavior of ionospheric delay (WoIC) and the improved results (WIC) for a 24-hour period. The maximum ionosphere effects occur, as expected, at 17 hours GPS time (@14 hours local time).

\subsection{Relative positioning test}

Further experiments were carried out in order to analyze the implemented model, considering now relative positioning. Baselines of approximately $100 \mathrm{~km}$ were processed in relation to the UEPP station. All RBMC stations participated in the estimation of the parameters of the model.

Results of the processing carried out using dual frequency receivers (Ashtech ZXII) were considered as "ground truth". 

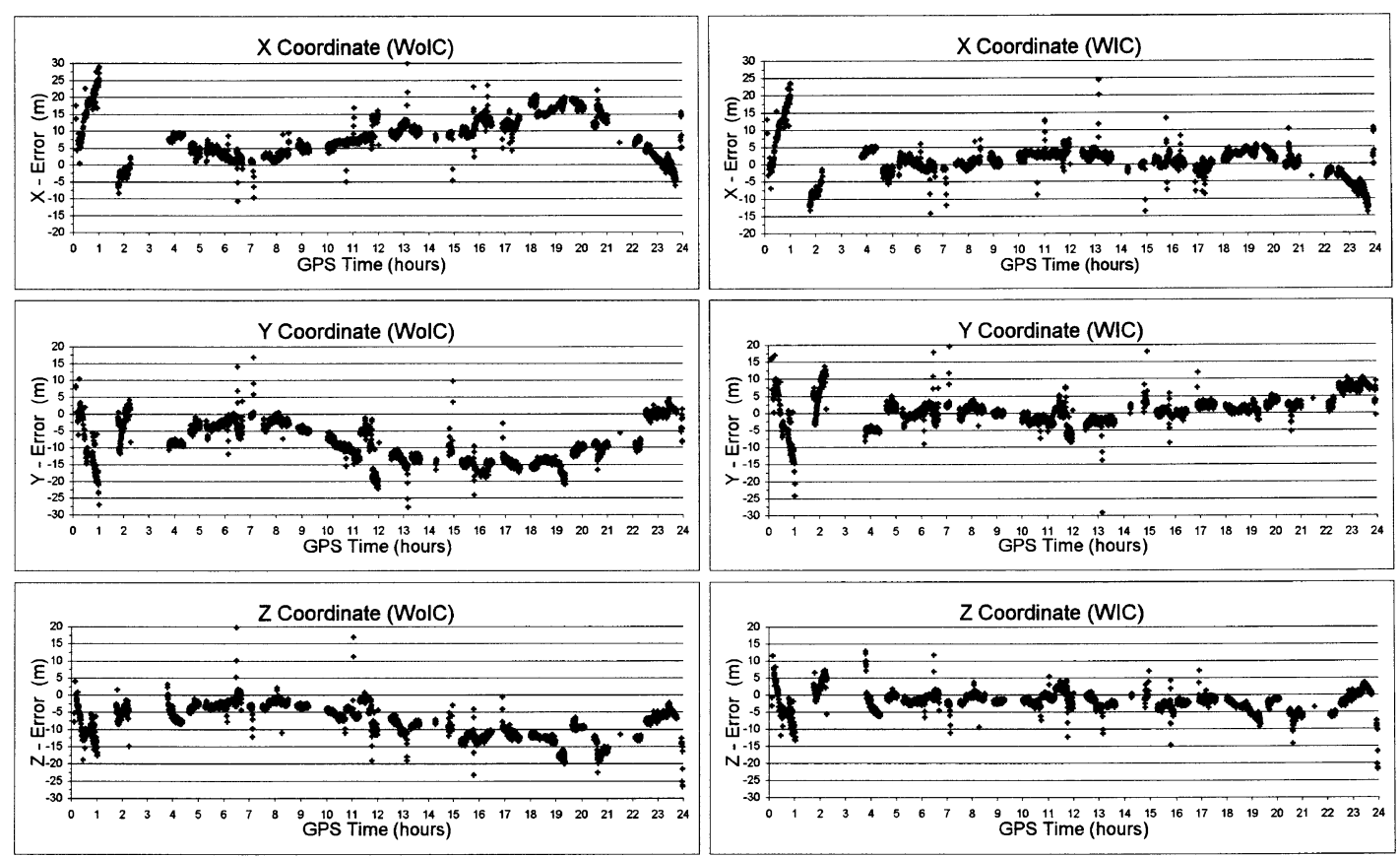

Fig. 7. An epoch per epoch solution (274/1998)_discrepancies between the "true" and the estimated cartesian coordinates of UEPP station (WoIC and WIC refer to the solution without and with ionospheric corrections).

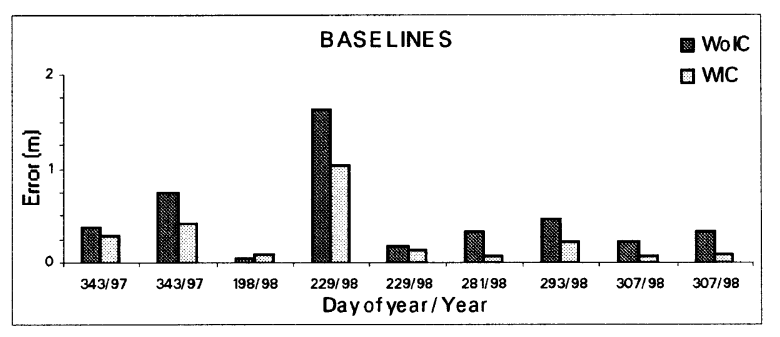

Fig. 8. Discrepancies between the "true" and the estimated distances (WoIC and WIC refer to the solution without and with ionospheric corrections).

For such cases, the double difference ionospheric free linear combination (ion free) was used as basic observable. The processing was carried out using the GPSurvey Software.

Again, two kinds of processing were carried out: WoIC and WIC. In both cases, IGS precise ephemerides and the Hopfield tropospheric model were used. Only observables with elevation angle larger than 15 degrees were used.

Figure 8 shows the discrepancies in distances between the baselines processed with ion free and L1 WoIC and L1 WIC.

From Fig. 8 one may conclude that there was an error reduction of the order of $50 \%$ after corrections were applied.

\section{Conclusion and Future Work}

A model for computing the effects of the ionosphere was presented. The experiments showed that this model provides an error reduction of $80 \%$, in relation to the point positioning carried out without correction. Metric accuracy was achieved for most cases. Considering an epoch per epoch solution, the results showed that the model improves the results even during the daily ionosphere maximum (17 hours GPS time $\approx 14$ hours local time).
For relative positioning the improvement was $50 \%$ for baselines of approximately $100 \mathrm{~km}$. Further studies related to improvement of L1 relative positioning should be performed, trying to identify the main drawbacks in such cases.

Additionally, new functions to model the ionospheric delay should be tested, such as spherical harmonic and polynomial. Besides this, tests will be accomplished considering the variation of the ionospheric shell height, taking into account both the temporal and spatial variability of the ionosphere in the equatorial region. Also, tests with data that will be collected during the period of maximum solar activity, ionospheric scintillation and magnetosphere substorm event will be accomplished. It is also our aim to produce ionospheric maps representing the ionospheric L1 carrier delay or TEC, for South America Region.

Considering the variability of the ionosphere in the equatorial region, it is recommended to study other zenith mapping functions to project the line-of-sight ionosphere delay into the vertical and to analyze the impact of ionospheric mapping function used in the proposed approach.

Acknowledgments. This work was developed with financial support of FAPESP (contract 95/08775-1) and CAPES/PICD.

\section{References}

Camargo, P. O., Modelo Regional da Ionosfera para uso em Posicionamento com Receptores de uma Frequiência, Ph.D. Thesis, Universidade Federal do Paraná, 191 pp., 1999.

Campos, M. A., L. Wanninger, and G. Seeber, Condições ionosféricas perturbadas e os sinais GPS, 3o. Congresso Internacional da Sociedade Brasileira de Geofísica, Rio de Janeiro, pp. 601-604, 1993.

Georgiadiou, Y., Modeling the ionosphere for an active control network of GPS stations, in LGR-Series - Publications of the Delft Geodetic Computing Centre, Delft University of Technology, n. 7, 1994.

Georgiadiou, Y. and A. Kleusberg, On the effects ionospheric delay on geodetic relative GPS positioning, Manuscripta Geodaetica, 13(1), 1-8, 
1998.

Jin, X. X., Theory of carrier adjusted DGPS positioning approach and some experimental results, Ph.D. Thesis, Delft University of Technology, 162 pp., 1996.

Klobuchar, J. A., Ionospheric time-delay algorithm for single-frequency GPS users, IEEE Transactions on Aerospace and Electronic Systems, AES-23(3), 325-331, 1987.

Klobuchar, J. A., Ionospheric effects on GPS, GPS World, April, 48-50, 1991.

Leick, A., GPS Satellite Surveying, 560 pp., John Wiley \& Sons, New York, 1995.

Monico, J. F. G., High Precision GPS Inter-continental Networks, Ph.D. Thesis, University of Nottingham, 205 pp., 1995.

Newby, S. P. and R. B. Langley, Three alternative empirical ionospheric models - are they better than GPS broadcast model?, in Proceedings of the Sixth International Geodetic Symposium on Satellite Positioning, pp. 240-244, Columbus, 1992.

NRCan, User's Guide-GPSPACE (GPS Positioning from ACS Clocks and Ephemerides-Version 3.2), Canadian Active Control System Operations, Geodetic Survey Division, Geomatics Canada, Natural Resources Canada, 1997.

Sardon, E. and N. Zarraoa, Estimation of total electron content using GPS data: how stable are the differential satellite and receiver instrumental biases, Radio Sci., 32, 1899-1910, 1997.

Teunissen, P. J., Quality control in geodetic networks, in Optimization and Design of Geodetic Networks, pp. 526-547, Berlin, 1985.

Wanninger, L., G. Seeber, and M. A. Campos, Use of GPS in the south of Brazil under severe ionospheric conditions, in IAG Symposium III, 10 pp., Heidelberg, 1991.

Wanninger, L., G. Seeber, and M. A. Campos, Limitations of GPS in equatorial regions due to the ionosphere, VII Simpósio Brasileiro de Sensoriamento Remoto, 14 pp., Curitiba, 1993.

Wells, D., N. Beck, D. Delikaraoglou, A. Kleusberg, E. J. Krakiwsky, G. Lachapelle, R. B. Langley, M. Nakiboglu, K. P. Schwarz, J. M. Tranquilla, and P. Vanicek, Guide to GPS Positioning, Canadian GPS Associates, Fredericton, New Brunswick, Canada, 1986.

Wilson, B. D., C. H. Yinger, W. A. Feess, and C. Shank, New and improved the broadcast interfrequency biases, GPS World, September, 56-66, 1999.

Zhong, D., Robust estimation and optimal selection of polynomial parameter for the interpolation of GPS heights, Journal of Geodesy, 9(71), 552-561, 1997.

P. O. Camargo (e-mail: paulo@prudente.unesp.br), J. F. G. Monico (e-mail: galera@prudente.unesp.br), and L. D. D. Ferreira (e-mail: danilo@geoc.ufpr.br) 